\title{
An Indefinite Cycle Traffic Light Timing Strategy
}

\author{
Ping Guo, Daiwen Lei, Lian Ye \\ College of Computer Science \\ Chongqing University \\ Chongqing,400044 \\ China
}

\begin{abstract}
Intelligent transportation signal control plays an important role in reducing traffic congestion and improving road capacity. The key of signal control is to adjust the traffic lights appropriately according to the traffic flow, which is an adaptive control. In this paper, we propose a new timing strategy. This strategy includes green time optimization and lane combination calculation. According to the real-time traffic flow, we optimize green time and calculate lane combination to adjust the cycle and then we can get the timing plan. The simulation results of random data and actual traffic data show that the strategy we proposed can increase traffic efficiency by more than $15 \%$ at intersections, reduce vehicle detention, and relieve traffic congestion.
\end{abstract}

Keywords-Traffic light; timing strategy; adaptive control; signal control; intelligent transportation

\section{INTRODUCTION}

With the development of the economy and the expansion of the city scale, the rapid growth of urban car has caused many problems such as traffic congestion and environmental pollution. The intelligent transportation system provides effective support for solving traffic congestion. It integrates multiple disciplines in the field of information and aims to establish a full-coverage, real-time, accurate, and efficient integrated transportation and management system [1], [2].

In recent years, the research on intelligent transportation systems has achieved many results. Collotta $\mathrm{M}$. proposed a novel approach to dynamically manage the traffic lights cycles and phases in an isolated intersection [3]. This method is a traffic lights dynamic control system that combines Wireless Sensor Network (WSN) for real-time traffic monitoring with multiple fuzzy logic controllers. This system outperforms other solutions in the literature, since it significantly reduces the vehicles waiting times. Li C. focused on an optimum route search in the in-vehicle routing guidance system. For the dynamic route guidance system (DRGS), it should provide dynamic routing advice based on real-time traffic information and traffic conditions, such as congestion and roadwork [4]. Darwish T. S. J. applied big data to intelligent transportation and proposed a real-time ITS (intelligent transportation system) big data analysis method for vehicle internet environment [5]. The Intelligent Transportation Project RoadEye was proposed to solve various traffic problems and make traffic safer. The project was able to detect weather conditions, maintain a safe distance and fixed speeds between vehicles [6]. Hassouneh Y. [7] proposed an ITS that can adapt its behavior in response to environmental changes. Cao Z. proposed a pheromone-based traffic management framework to reduce traffic congestion and unify dynamic vehicle routing and traffic control strategies [8].
The intersection is the node that transforms the traffic flow and plays an important role in the road network [9]. The control of traffic lights at intersections is the basis and guarantee for efficient and orderly operation of urban traffic. The merits of signal control methods directly determine the smoothness of traffic flow at intersections [10]. Traffic light control [11] at the intersection mainly includes timing control, induction control and adaptive control [12]. The timing control method is based on the use of a fixed green light time in each phases, which is simple and easy to maintain. Induction control is to set up a vehicle detector on the entrance of the intersection, and the traffic light timing plan changes with traffic flow in real time. This control is more suitable for situations where traffic flow at intersections is not obvious, saturation is not high, or the traffic flow in each phase is quite different. Adaptive control is to collect the traffic flow information in all directions of the intersection in real time, and calculate the green light time according to the prediction model to adapt to the change of intersection traffic. Its advantage is that it can reduce the delay time of vehicles and improve the communication efficiency of vehicles at intersections.

There are about 20 kinds of common traffic light control systems. They include OPAC (Optimized Policies for Adaptive Control) proposed by Nathan Gartner [13], [14], SCOOT (Split Cycle Offset Optimization Technique) proposed by the Transport Research Institute of the United Kingdom [15], SCATS (Sydney Coordinated Adaptive Traffic System) proposed by Road Traffic Bureau of New South Wales, Australia [16] and RHODESReal-time, Hierarehieal,Optimized, Distributed and Effective Systemdeveloped by the University of Arizona [17].

The key of intersection traffic light control is the traffic light timing calculation. Gttlich $\mathrm{S}$. proposed the use of the traffic flow conservation law to calculate the optimal traffic light timing plan at the intersection [18]. Younes M. B. designed an efficient dynamic traffic light timing algorithm that adjusts the optimal green time for each traffic flow based on the real-time traffic situation around the intersection. The algorithm also takes into account the presence of emergency vehicles so that they can quickly pass through the intersections [19].

In this paper, based on adaptive control, a single intersection traffic light timing model is studied and a set of timing plan calculation algorithm is proposed. Simulation experiments show that the proposed timing plan can improve the traffic efficiency at the intersection by about $15 \%$ to $20 \%$. The rest of the paper is organized as follows: Section II describes the intersection model and some basic assumptions of this paper; Section III presents a set of timing strategies with indefinite 
cycle and indefinite phases; Simulation experiments and results analysis are given in Section IV. Finally, the paper is concluded in Section V.

\section{Assumptions AND DEFINITIONS}

The intersection is the point of convergence between people and vehicles. People and vehicles from different directions arrive at their destinations through intersections, which determines the importance and complexity of traffic control at intersections in traffic systems. A typical intersection model is shown in Fig. 1. To simplify the discussion, we assume that

1) The right turn of the vehicle is not restricted by the traffic light and it is immediately released.

2) Do not consider pedestrians' influence on traffic.

3) The lane $L_{i}(1 \leq i \leq 8)$ can have different travel sequences and green light time during an intersection signal cycle.

4) At most two lanes of vehicles are allowed to travel at the same time.

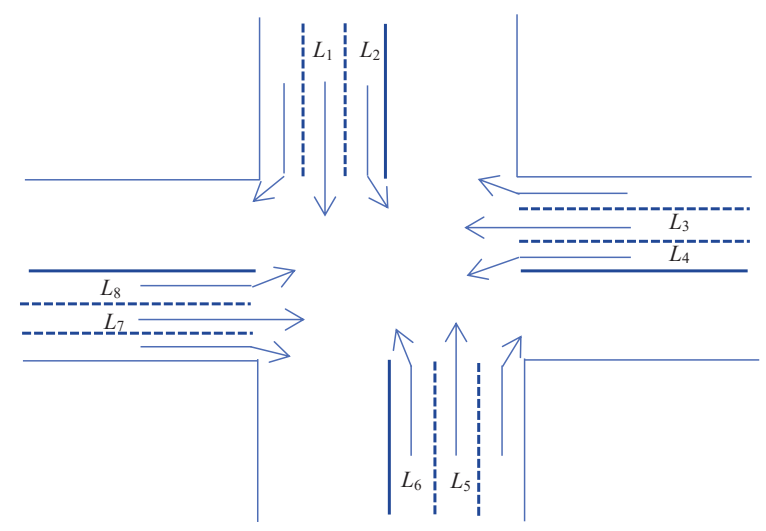

Fig. 1. Intersection lane layout.

The combination of any two lanes that do not have collisions (that is, no cross traffic) is called a phase, and the 8 lanes in Fig. 1 can make up to 12 phases. For example, $L_{1}$ and $L_{2}$ may form one phase, and $L_{1}$ and $L_{5}$ may also form one phase.

For the convenience of description in this paper, we give the following definitions:

Definition 1. As for any lane $L_{i}$, when lane $L_{j}(j \neq i)$ does not affect the traffic of lane $L_{i}, L_{j}$ is called $L_{i}$ 's compatible lane. Each lane has multiple compatible lanes. For example, lane $L_{6}$ have compatible lanes: $L_{2}, L_{3}, L_{5}$.

Definition 2. In two lanes of a phase, the lane first determined the right to travel is called the main lane.

Definition 3. The traffic rate refers to the number of vehicles passing through the unit time. Obviously, the more vehicles that pass during the unit time, the higher the traffic rate is. The traffic rate for each lane is denoted by $q_{i}(i=1,2, \cdots, 8)$, and the traffic rate of the intersection is denoted by $q_{0}$.
Definition 4. In all lanes, the maximum number of vehicles that pass through the intersection without stagnating during the unit time is called intersection saturation flow $q$ [20].

Obviously, saturation flow is greater than or equal to the traffic rate. When the traffic rate reaches saturation flow, the traffic rate is the highest. In this paper, we assume that the saturation flow of the lane is the same as the saturation flow of the intersection.

In practical applications, the time spent by different types of vehicles passing through the intersection is not the same. For ease of calculation and comparison, we use Table I to convert all types of vehicles into standard vehicle. The vehicles described later in this article refers to the standard vehicle.

TABle I. Vehicle Classification and Vehicle Conversion FACTOR

\begin{tabular}{llll}
\hline Vehicle Type & $\begin{array}{l}\text { vehicle } \\
\text { conversion factor }\end{array}$ & Load and power & Explanation \\
\hline Small trucks & 1.0 & $\begin{array}{l}\text { Load quality } \leq 2 \\
\text { tons }\end{array}$ & - \\
Medium truck & 1.5 & $\begin{array}{l}2 \text { tons }<\text { load qual- } \\
\text { ity } \leq 7 \text { tons } \\
7 \text { tons }<\text { load }\end{array}$ & Including cranes \\
Large trucks & 2.0 & $\begin{array}{l}\text { quality } \leq 14 \text { tons } \\
\text { Load quality }>14\end{array}$ & - \\
Heavy-duty truck & 3.0 & $\begin{array}{l}\text { tons } \\
-\end{array}$ & $\begin{array}{l}\text { Including } \\
\text { trailer,flatbed } \\
\text { trailer }\end{array}$ \\
Trailer & 3.0 & & - \\
& & - & - \\
Container car & 3.0 & $\begin{array}{l}\text { Rated seat } \leq 19 \\
\text { seats }\end{array}$ & - \\
small-sized buses & 1.0 & $\begin{array}{l}\text { Rated seat }>19 \\
\text { seats }\end{array}$ & - \\
large-sized buses & 1.5 & - & - \\
motorcycle & $0.4-0.6$ & - &
\end{tabular}

\section{TIMING StRATEGY}

\section{A. Cycle and Traffic Light Control}

Intersection traffic light cycle (hereinafter referred as cycle) is an important parameter of the timing strategy. Short cycles can easily cause the traffic lanes to alternate too frequently, affecting traffic flow through the intersections and excessively long cycles lead to increased waiting time for vehicles, causing a backlog of vehicles. Therefore, choosing an appropriate cycle is important for improving traffic efficiency.

Due to the uncertainty of the vehicle arrival time at the intersection, we use the traffic rate as the goal to calculate the green light time of each lane and cycle. The lower limit of the green light time is $T_{g}$, and the upper limit of the cycle is $T_{\max }$. Thus, the signal control strategy is:

1) In each cycle, each lane has a green light right to travel;

2) The green light time of each lane is not less than $T_{g}$, and the traffic sequence is not fixed;

3) Each cycle time is not fixed, but not greater than $T_{\max }$;

4) Only vehicles of two compatible lanes are allowed to pass at the same time.

In order to implement the above strategy and facilitate control, the vehicle traffic data collected in the $i$-th cycle will be calculated in the $(i+1)$ th cycle to form a timing plan, which is implemented in the $(i+2)$ th cycle. This means that 
the vehicle arrival data of the $(i+2)$ th cycle is predicted by the traffic data of the $i$-th cycle, and the efficiency of the $(i+2)$ th cycle is improved by adjusting the timing plan.

\section{B. Timing Calculation}

The timing calculation is to give the green light time and traffic sequence of each lane in a cycle.

1) Green time optimization: Assume that the green light time of lane $L_{j}(j=1,2, \cdots, 8)$ in the $i$-th cycle is $T_{j}{ }^{i}$ and the number of vehicles passing through the intersection is $S_{j}{ }^{i}$. The traffic rate of lane $L_{j}$ is $q_{j}{ }^{i}=\frac{S_{j}{ }^{i}}{T_{j}{ }^{i}}$.

If the number of vehicle arrivals in the $(i+2)$ th cycle is the same as the $i$-th cycle, the traffic rate $q_{j}{ }^{i+2}$ can be increased by calculating the green light time $T_{j}{ }^{i+2}$ of the $(i+2)$ th cycle in (1).

$$
T_{j}{ }^{i+2}= \begin{cases}\frac{S_{j}{ }^{i}}{\mu q_{i}}, & q_{j}{ }^{i} \geq \mu q \\ \frac{S_{j}{ }^{i}}{\lambda q}, & q_{j}{ }^{i} \leq \lambda q \\ T_{j}{ }^{i}, & \lambda q<q_{j}{ }^{i}<\mu q\end{cases}
$$

In (1), to make the green light time robust, the parameters $\mu(0<\mu \leq 1)$ and $\lambda(0<\lambda<1, \lambda<\mu)$ are set to limit the adjustment of the green light time:

(a) when $\lambda q<q_{j}{ }^{i}<\mu q$, set $T_{j}{ }^{i+2}=T_{j}{ }^{i}$. That is, do not adjust the green light time.

(b) when $q_{j}{ }^{i} \geq \mu q$, it means that there are too many vehicles in the lane $L_{j}$ during ith cycle and the green light time is relatively short, and it is necessary to increase the green light time to allow more vehicles to pass.

(c) when $q_{j}{ }^{i} \leq \lambda q$, it means that there are few vehicles in the lane $L_{j}$ during ith cycle and the green time is longer. And the green light time needs to be shortened to increase the traffic rate of the lane. In order to ensure that each lane has the right to travel during the cycle, the minimum green light time is $T_{g}$.

To facilitate the operation of the control system, the green light time after the optimization of (1) is adjusted to a multiple of 5. This gives the predicted green light time $T_{j}{ }^{i+2}$ $(1 \leqslant j \leqslant 8)$ for the $(i+2)$ th cycle.

2) Lane combination calculation: The green light time obtained in the previous section is considered from the perspective of the traffic of each lane. Vehicle traffic at intersections also needs to consider the cycle and the traffic sequence in order to obtain a timing plan that can be implemented.

In the timing plan, the traffic sequence at the intersection is called the lane combination. A lane combination consists of two lane sequences, as in (2).

$$
\Pi=\left\{\begin{array}{l}
L_{i 1} L_{i 2} \cdots L_{i k} \\
L_{j 1} L_{j 2} \cdots L_{j k}
\end{array}\right.
$$

In $\Pi, L_{i p}, L_{j q} \in\left\{L_{1}, L_{2}, \cdots, L_{8}\right\}, L_{i p}$ and $L_{j p}$ are mutually compatible lanes, which belong to lane sequence one and lane sequence two, respectively. And they all travel at the same time. Remark $\mathrm{R}\left(L_{i}\right)$ is the remaining green time of the lane $L_{i}$. The calculation algorithm of the lane combination is as follows:

(a) If every lane has obtained the right to travel, then stop.

(b) Randomly select a lane $L_{m}$ that has not obtained the right of travel as the main lane and give it the right to travel.

(c) If there are $L_{m}$ compatible lanes that do not obtain the right of travel, then randomly select a lane $L_{i}$ to give it the right to travel with the main lane $L_{m}$ at the same time. Therefore $L_{i}$ and $L_{m}$ travel simultaneously and form a lane combination segment $\left(\begin{array}{c}L_{m} \\ L_{i}\end{array}\right)$, and then go to step(d); otherwise, $L_{m}$ travel alone and form a lane combination $\left(\begin{array}{c}L_{m} \\ 0\end{array}\right)$, then go to step(a).

(d) If $\mathrm{R}\left(L_{m}\right)=\mathrm{R}\left(L_{i}\right)$, then go to $\operatorname{step}(\mathrm{a})$.

(e) If $\mathrm{R}\left(L_{m}\right)>\mathrm{R}\left(L_{i}\right)$, let $\mathrm{R}\left(L_{m}\right) \leftarrow \mathrm{R}\left(L_{m}\right)-\mathrm{R}\left(L_{i}\right)$ and $L_{m}$ as main lane; otherwise, let $\mathrm{R}\left(L_{i}\right) \leftarrow \mathrm{R}\left(L_{i}\right)-\mathrm{R}\left(L_{m}\right)$ and $L_{i}$ as main lane. Then go to $\operatorname{step}(\mathrm{c})$.

Obviously, in the above algorithm, different choices of $L_{m}$ and $L_{i}$ will result in different lane combinations. The time spent traveling in the traffic sequence is called the cycle of lane combination, and the smallest cycle of the lane combination recorded as $T_{\min }$. When $T_{\min } \leqslant T_{\max }$, all lane combinations with a $T_{\min }$ cycle are called candidate lane combinations; otherwise, the green time of the lane is compressed as described below until $T_{\min } \leqslant T_{\max }$.

(a) Randomly select a lane combination with a cycle $T_{\min }$;

(b) Calculate the difference between $T_{\min }$ and $T_{\max }$, then we can get the current time needed to compress:

$$
\Delta T=T_{\min }-T_{\max }
$$

(c) In $\Pi$, the required compression time for each lane is according to the ratio of the initial traffic rate of the lane. That is:

$$
T_{i}=\Delta T-\Delta T * \frac{q_{i}}{q_{1}+q_{2}+q_{3}+\cdots+q_{8}}
$$

3) Lane traffic plan: In the candidate lane combinations calculated in 2) of the part B above, there may be multiple kinds of lane combinations. There may be 4 pairs, 3 pairs, 2 pairs, and 1 pair that the green time of main lanes and compatible lane ends at the same time. In the actual traffic light control at intersection, the green light time of the two lanes in the same phase, ends at the same time. Therefore, in the candidate lane combination, the lane combination having the most pairs that the green time of main lanes and compatible lane ends at the same times is selected as the implemented lane traffic plan.

For example, if the optimized green light time of lanes $L_{1}$ to $L_{8}$ is $35 \mathrm{~s}, 15 \mathrm{~s}, 35 \mathrm{~s}, 15 \mathrm{~s}, 30 \mathrm{~s}, 15 \mathrm{~s}, 20 \mathrm{~s}$ and $10 \mathrm{~s}$ respectively, we can get two lane combinations:

$$
\begin{gathered}
\Pi_{1}=\left\{\begin{array}{l}
L_{5} L_{2} L_{2} L_{8} L_{6} L_{4} \\
L_{1} L_{1} L_{7} L_{7} L_{3} L_{3}
\end{array}\right. \\
\Pi_{2}=\left\{\begin{array}{l}
L_{2} L_{5} L_{5} L_{3} L_{3} L_{3} L_{8} \\
L_{1} L_{1} L_{6} L_{6} L_{4} L_{7} L_{7}
\end{array}\right.
\end{gathered}
$$

In $\Pi_{1}$ there are two pairs of compatible lanes ( $L_{8}$ and $L_{7}$, $L_{4}$ and $L_{3}$ ) that green time ends at the same time. And in $\Pi_{2}$, 
there is only one pair of compatible lanes $\left(L_{8}\right.$ and $\left.L_{7}\right)$ that green time ends at the same time. So we choose $\Pi_{1}$ as the implemented lane traffic plan.

\section{EXPERIMENTS AND ANALYSIS}

In this section, simulations of the timing strategies proposed in this paper are carried out by using the vehicle's simulation data and measured data at intersections.

\section{A. Experimental Environment}

The environment for the simulation experiments in this paper is as follows:

(a) hardware configuration:

(i) processor: Inter(R) $\quad$ Core $(\mathrm{TM}) \quad \mathrm{i} 5-2400 \quad \mathrm{CPU}$ @ 3.10GHZ

(ii) RAM: $8.00 \mathrm{G}$

(b) related Software

(i) operating system: Windows 10

(ii) software tools: Microsoft matlab2014

(iii) programming language: $\mathrm{c}++$

\section{B. Experimental Data}

The simulation data of this paper is divided into two categories: the first group of vehicle arrival data is randomly generated, and the second group of vehicle arrival data is real data we measured.

(a) Random data

In each cycle, suppose that the number of arriving vehicles $S_{i}$ in lane $L_{i}$ is satisfied with the inequality (5).

$$
0 \leq S_{i} \leq q * T_{i}
$$

where $T_{i}$ is the green time of lane $L_{i}$. This assumption guarantees that there is no stranded vehicle in each lane at the end of the cycle.

(b) Measured data

We use the real data as vehicle arrival data. Assume that the vehicle arrives randomly at an intersection and obeys a Poisson distribution (6). The arrival rate is the average arrival rate in the measured data sample.

$$
P(k)=\frac{(\rho k)_{t} e^{-(\rho t)}}{k !}=\frac{m^{k} e^{-m}}{k !}
$$

Where $\rho$ denotes the average vehicle arrival rate (vehicles/second) and $m=\rho t$ denotes the average number of vehicles arriving within time $t$.

In order to facilitate calculation and comparison, the actual measured data is converted into standard vehicles according to Table I, as shown in Table II. The vehicle arrival rate is shown in Table III.

TABLE II. The Total Number of Standard Vehicles at INTERSECTIONS

\begin{tabular}{lllllllll}
\hline Time & $L_{1}$ & $L_{2}$ & $L_{3}$ & $L_{4}$ & $L_{5}$ & $L_{6}$ & $L_{7}$ & $L_{8}$ \\
\hline $7: 30-7: 45$ & 87 & 56.5 & 306.5 & 144 & 85.5 & 63.5 & 379.5 & 73 \\
$7: 45-8: 00$ & 114 & 44.5 & 332 & 129.5 & 100.5 & 83 & 313 & 57 \\
$8: 00-8: 15$ & 86 & 41.5 & 343 & 101.5 & 77 & 48.5 & 284.5 & 50 \\
$8: 15-8: 30$ & 79.5 & 36.5 & 304 & 102.5 & 54 & 46.5 & 276.5 & 60 \\
\hline
\end{tabular}

TABLE III. Measured Vehicle Arrival Rate

\begin{tabular}{lllllllll}
\hline Time & $L_{1}$ & $L_{2}$ & $L_{3}$ & $L_{4}$ & $L_{5}$ & $L_{6}$ & $L_{7}$ & $L_{8}$ \\
\hline 7:30-7:45 & 0.097 & 0.063 & 0.341 & 0.160 & 0.095 & 0.069 & 0.422 & 0.081 \\
7:45-8:00 & 0.127 & 0.049 & 0.369 & 0.214 & 0.112 & 0.092 & 0.348 & 0.063 \\
8:00-8:15 & 0.096 & 0.046 & 0.392 & 0.113 & 0.086 & 0.054 & 0.316 & 0.056 \\
8:15-8:30 & 0.088 & 0.041 & 0.338 & 0.114 & 0.060 & 0.052 & 0.307 & 0.067 \\
\hline
\end{tabular}

\section{Experimental Results}

We compared the timing plan proposed in this paper (abbreviated as ITP, Improved timing plan) and the fixed time plan (FTP) through the simulation experiments. Experimental results are evaluated using the following indicators:

(a) Number of traffic vehicles: The sum of the number of traffic vehicles in each lane during a cycle.

(b) Traffic efficiency: The ratio of number of traffic vehicles to cycle.

(c) Number of detention vehicles: The sum of the number of vehicles staying in each lane after the end of the cycle.

1) Random data simulation results analysis: Simulation experiments conducted 50 cycles. In the first cycle, it is assumed that the green time of lanes $L_{1}$ to $L_{8}$ are: $30 \mathrm{~s}, 15 \mathrm{~s}$, $30 \mathrm{~s}, 15 \mathrm{~s}, 30 \mathrm{~s}, 15 \mathrm{~s}, 30 \mathrm{~s}, 15 \mathrm{~s}$. The number of arrival vehicles is calculated according to (5), and the lane combination is:

$$
\left\{\begin{array}{l}
L_{1} L_{2} L_{3} L_{4} \\
L_{5} L_{6} L_{7} L_{8}
\end{array}\right.
$$

From second to the 50th cycle, the ITP is the timing plan described in Section III. The number of arrival vehicles in each lane is still calculated according to (5). The FTP still adopts the first cycle timing plan, and the number of arrival vehicles in each lane is the same as the ITP timing plan. Other assumptions for the ITP: $T_{\max }=90$ seconds, $\mathrm{q}=0.5$ (vehicles/second), $\lambda=0.4, \mu=0.7, T_{g}=10$ seconds. The simulation results are shown in Fig. 2 and 3.

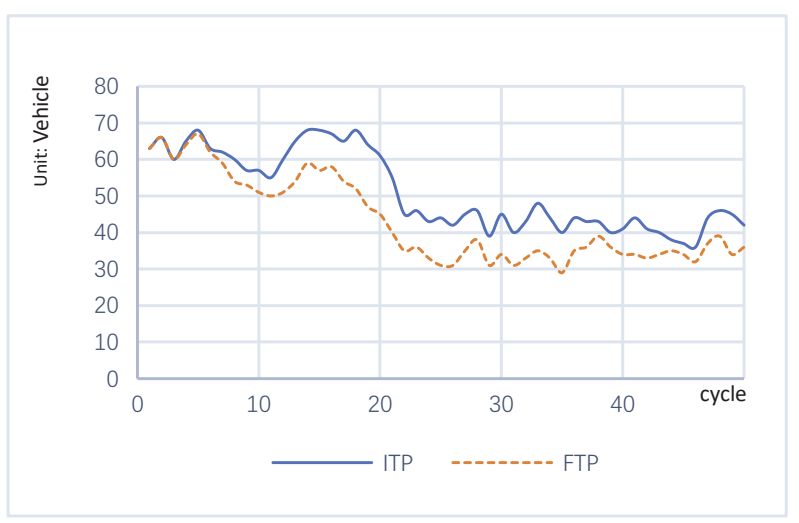

Fig. 2. Number of traffic vehicles comparison.

From Fig. 2, we can see that after several cycle adjustments, the number of traffic vehicles in the timing plan proposed in this paper is significantly increased compared to the number of vehicles in fixed timing plan. And the average number of the traffic vehicles increased by $20.3 \%$. In the adjustment process, there are some cycles time in ITP are 


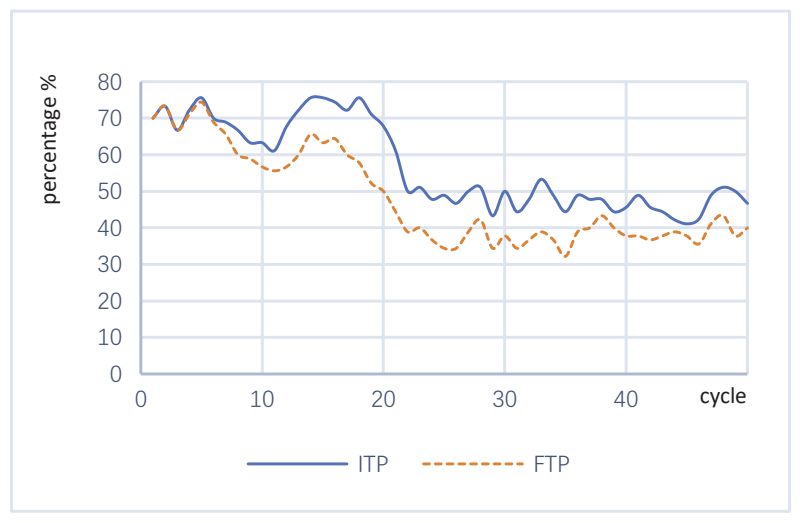

Fig. 3. Traffic efficiency comparison.

smaller than $T_{\max }$. This will make the cycle change faster and make vehicles wait less time, then traffic efficiency is improved. From Fig. 3, traffic efficiency increased by an average of $19.9 \%$. This shows that the timing strategy proposed in this paper significantly improves the traffic efficiency.

2) Measured data simulation results analysis: The simulation experiment is conducted for 1 hour. In the first cycle, it is assumed that the green light time of lanes $L_{1}$ to $L_{8}$ are: 30 s, $15 \mathrm{~s}, 30 \mathrm{~s}, 15 \mathrm{~s}, 30 \mathrm{~s}, 15 \mathrm{~s}, 30 \mathrm{~s}, 15 \mathrm{~s}$. The number of arrival vehicles is the measured data in Table III and the lane combination is:

$$
\left\{\begin{array}{l}
L_{1} L_{2} L_{3} L_{4} \\
L_{5} L_{6} L_{7} L_{8}
\end{array}\right.
$$

From the second to the 50th cycle, the number of arrival vehicles in each lane is obtained from Table III. The FTP still adopts the first cycle timing plan, and the number of arrival vehicles in each lane is the same as the ITP. Parameter settings for the ITP: $T_{\max }=90$ seconds, $q=0.5$ (vehicles/second), $\lambda$ $=0.4, \mu=0.7, T_{g}=10$ seconds. The simulation results are shown in Fig. 4, 5 and 6.

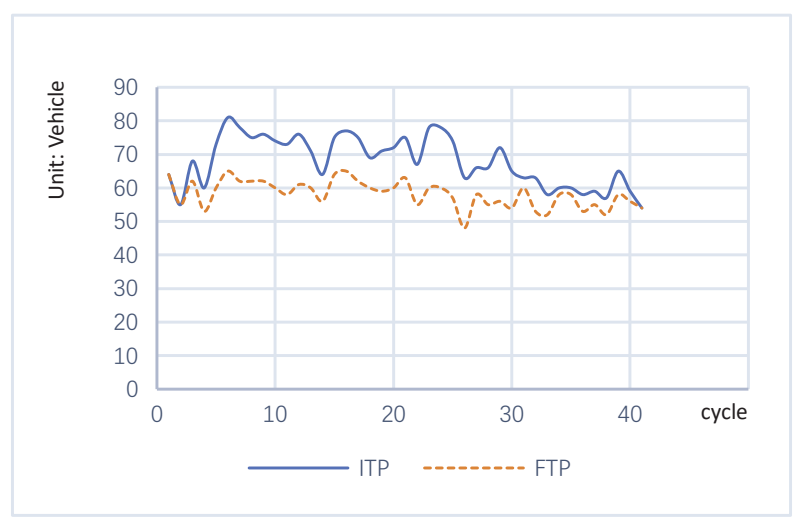

Fig. 4. Number of traffic vehicles comparison.

From Fig. 4, after several cycle adjustments, the number of traffic vehicles in ITP is higher than the number of traffic vehicles in FTP. And the average number of traffic vehicles

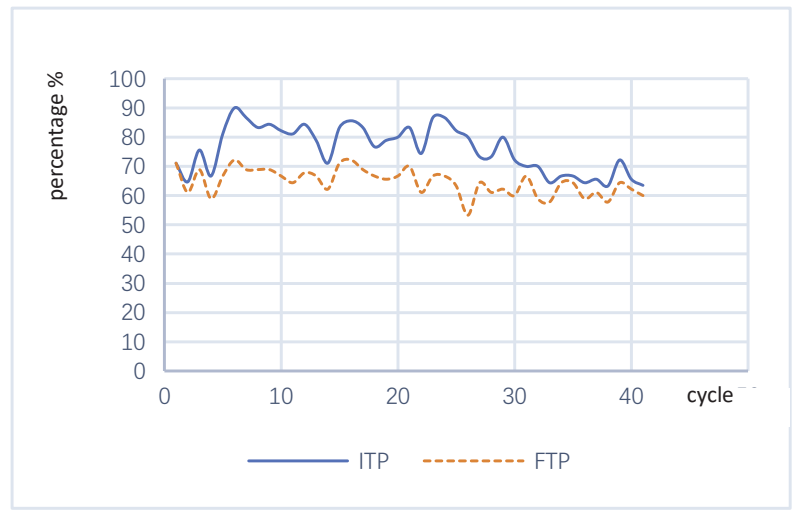

Fig. 5. Traffic efficiency comparison.

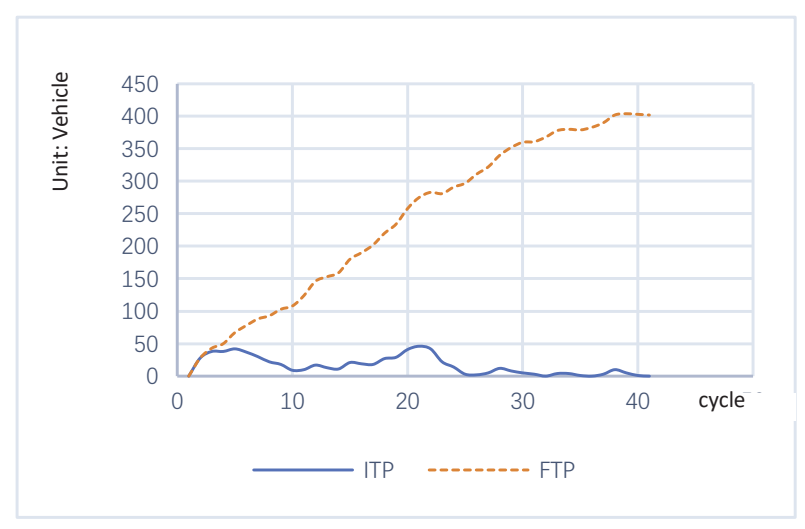

Fig. 6. Comparison of the number of retained vehicles.

increased by $16.6 \%$. At the same time, from Fig. 5 , the traffic efficiency in one hour increased by an average of $17.2 \%$. It can also be seen from the Table III that the traffic rate of $L_{3}$ and $L_{7}$ are relatively large. When ITP is adopted, the green light time of large traffic rate lane will be appropriately extended, and the green light time of small traffic rate lane will be reduced to a sufficient extent. As shown in Fig. 6, the gap between the number of retained vehicles in ITP and the number of retained vehicles in FTP is increasing.

In summary, after the adjustment of ITP for about 5 cycles, its superiority gradually emerged. The ITP is significantly better than the FTP in terms of the number of passing vehicles, the efficiency of traffic, and the number of retained vehicles.

\section{CONClusion}

We proposes an adaptive timing strategy IPT with indefinite cycle and indefinite phase. On the one hand, the strategy can automatically adjust the green light time of each lane according to the traffic flow collected in real time. So that the green light time of lanes with less vehicle or no car is shortened, and the green light time of lanes with more cars is longer. On the other hand, the strategy calculates the appropriate lane combination through the concept of main lanes, compatible lanes, etc. to facilitate traffic light control at intersections. The 
ITP improves the traffic efficiency of vehicles at intersections by increasing the utilization of the green light. The simulation experiment results show that the ITP increases the traffic efficiency compared to the traditional fixed timing plan by an average of $15 \%$ to $20 \%$. The use of the ITP timing strategy can effectively shorten the waiting time of the vehicle, reduce the vehicle stagnation and improve the traffic condition of the intersection.

For further work, firstly, when the optimized cycle exceeds the maximum cycle, we can study the compression method so that the cycle satisfies both the cycle requirement and the maximum traffic rate. Secondly, in the lane combination calculation, we can consider the green time as the information of selecting the main lane, so that the implemented lane combination can be calculated more quickly.

\section{REFERENCES}

[1] Gang Xiong,Fenghua Zhu,Xiwei Liu,Xisong Dong,Wuling Huang,Songhang Chen,Kai Zhao. "Cyber-physical-social System in Intelligent Transportation". IEEE/CAA Journal of Automatica Sinica,2015,2(03):320-333.

[2] Li D,Deng L, Cai Z, et al. "Intelligent Transportation System in Macao based on Deep Self Coding Learning[J]. IEEE Transactions on Industrial Informatics,2018“, doi: 10.1109/TII.2018.2810291.

[3] Collotta M, Bello L L, Pau G. "A novel approach for dynamic traffic lights management based on Wireless Sensor Networks and multiple fuzzy logic controllers“. Expert Systems with Applications, 2015 42(13):5403-5415

[4] Li C, Anavatti S G, Ray T. "Analytical Hierarchy Process Using Fuzzy Inference Technique for Real-Time Route Guidance System“. IEEE Transactions on Intelligent Transportation Systems, 2014, 15(1):84-93.

[5] Darwish T S J, Bakar K A. "Fog Based Intelligent Transportation Big Data Analytics in The Internet of Vehicles Environment: Motivations, Architecture, Challenges and Critical Issues“. IEEE Access, 2018, doi: 10.1109/ACCESS.2018.281598

[6] Ibrahim M, Riad M, El-Abd M. "RoadEye The Intelligent Transportation System“. 2017 International Conference on Computer Systems and Applications (AICCSA), Hammamet, Tunisia, IEEE, 2017:21-22.

[7] Hassouneh Y, Tumar I and Abu-Issa A. "Case study about capturing uncertainty in adaptive intelligent transportation systems".2017 International Conference on Engineering and Technology (ICET), Antalya, Turkey, 2017:1-2.
[8] Cao Z, Jiang S, Zhang J, et al. "A Unified Framework for Vehicle Rerouting and Traffic Light Control to Reduce Traffic Congestion“. IEEE Transactions on Intelligent Transportation Systems, 2017, 18(7):19581973.

[9] Khelafa I, Ballouk A, Baghdad A, et al. "Development of control algorithm for urban traffic“. 2017 International Conference on Electrical and Information Technologies(ICEIT)ZhuhaiChina ,2017:1-5.

[10] Collotta M, Pau G, Scat G, et al. "A dynamic traffic light management system based on wireless sensor networks for the reduction of the redlight running phenomenon“. Transport \& Telecommunication Journal, 2014, 15(1):1-11.

[11] F. Faheem, Z. Zuraidah, A. Kayani and A. K. Aminuddin. "Optimization of vehicle actuation and multiplan algorithms for urban traffic control systems".2017 IEEE Conference on Systems, Process and Control (ICSPC), Malacca, Malaysia,2017: 59-64.

[12] Denisova, L.A. Meshcheryakov, V.A . "Automatic parametric synthesis of a control system using the genetic algorithm", Automation and Remote Control,2015,76(1):149156.

[13] Gartner N, Pooran F, Andrews C. "Optimized Policies for Adaptive Control Strategy in Real-Time Traffic Adaptive Control Systems: Implementation and Field Testing". Transportation Research Record Journal of the Transportation Research Board, 2002, 1811(1):148-156.

[14] Gartner N, Pooran F and Andrews C. "Implementation of the OPAC adaptive control strategy in a traffic signal network". 2001 IEEE Intelligent Transportation Systems, Oakland, CA, 2001: 195-200.

[15] Bretherton D, Bodger M, Baber N. "SCOOT - the future [urban traffic control]“. 12th IEE International Conference on Road Transport Information and Control, 2004. RTIC 2004. London, UK, 2004:301-306.

[16] Luk, J.Y.K.. "Two Traffic-responsive Area Traffic Control Methods: SCAT and SCOOT “. Traffic Engineering \& Control, 1984, 251:14-22.

[17] Mirchandani P, Wang F Y. "RHODES to intelligent transportation systems“. IEEE Intelligent Systems, 2005, 20(1):10-15.

[18] Gttlich S, Potschka A, Ziegler U. "Partial Outer Convexification for Traffic Light Optimization in Road Networks“. Siam Journal on Scientific Computing, 2017, 39(1):B53-B75.

[19] Younes M B, Boukerche A. "An efficient dynamic traffic light scheduling algorithm considering emergency vehicles for intelligent transportation systems“. Wireless Networks, 2017 DOI: 10.1007/s11276-017-14825 .

[20] Yunfeng Gao, Xiaoguang Yang, Hua Hu. "The theoretical model of basic saturation flow rate based on the car-following model ". 2005 Cross-Strait Intelligent Transportation System Seminar and Tongzhou Traffic ForumShanghaiChina2005:3-8. 